\title{
Some Issues on Consistency of Fuzzy Preference Relations ${ }^{1}$
}

\author{
E. Herrera-Viedma, F. Herrera, F. Chiclana, M. Luque \\ Dept. of Computer Science and Artificial Intelligence \\ University of Granada, 18071 - Granada, Spain \\ viedma,herrera@decsai.ugr.es,fchiclana@teleline.es, mluque@decsai.ugr.es
}

\begin{abstract}
In decision making, in order to avoid misleading solutions, the study of consistency when the decision makers express their opinions by means of preference relations becomes a very important aspect. In decision making problems based on fuzzy preference relations the study of consistency is associated with the study of the transitivity property. In this paper, a new characterization of the consistency property defined by the additive transitivity property of the fuzzy preference relations is presented. Using this new characterization a method for constructing consistent fuzzy preference relations from a set of n-1 preference data is proposed. Applying this method it is possible to assure better consistency of the fuzzy preference relations provided by the decision makers, and in such a way, to avoid the inconsistent solutions in the decision making processes. Additionally, a similar study of consistency is developed for the case of multiplicative preference relations.
\end{abstract}

Keywords: decision making, fuzzy preference relations, multiplicative preference relations, consistency, reciprocity.

\section{Introduction}

As it is known, most decision processes are based on preference relations, in the sense that processes are linked to some degree of preference of any alternative over another. The use of preference relations is usual in decision making $[2,6,11,15,16]$. Therefore, to establish properties to be verified by such preference relations is very important for designing good decision making models.

One of these properties is the so called consistency property. The lack of consistency in decision making can lead to inconsistent conclusions; that is why it is important, if not crucial, to study conditions under which consistency is satisfied $[5,6,11,16]$. On the other hand, perfect consistency is difficult to obtain in practice, specially when measuring preferences on a set with a large number of alternatives.

Clearly, the problem of consistency itself includes two problems $[4,8]$ :

(i) when an expert, considered individually, is said to be consistent and,

(ii) when a whole group of experts are considered consistent.

\footnotetext{
${ }^{1}$ Cite as: E. Herrera-Viedma, F. Herrera, F. Chiclana, M. Luque: Some Issues on Consistency of Fuzzy Preference Relations. European Journal of Operational Research 154(1), 98-109, April 2004. doi:10.1016/S03772217(02)00725-7
} 
In this paper we will focus on the first problem, assuming that expert's preferences are expressed by means of a preference relation defined over a finite and fixed set of alternatives.

In a crisp model, where an expert provides his/her opinion on the set of alternatives, $X=\left\{x_{1}, x_{2}, \ldots, x_{n} ; n \geq 2\right\}$, by means of a binary preference relation, $R$, the concept of consistency has traditionally been defined in terms of acyclicity [13], that is the absence of sequences such as $x_{1}, x_{2}, \ldots, x_{k}\left(x_{k+1}=x_{1}\right)$ with $x_{j} R x_{j+1} \forall j=1, \ldots, k$.

In a fuzzy context, where an expert expresses his/her opinions using fuzzy preference relations, a traditional requirement to characterize consistency is using transitivity, in the sense that if an alternative $x_{i}$ is preferred to alternative $x_{j}$ and this one to $x_{k}$ then alternative $x_{i}$ should be preferred to $x_{k}$. Stronger conditions have been given to define consistency, for example max-min transitivity property or additive transitivity property $[6,14,15,17]$. However, the problem is the difficulty to check and to guarantee such consistency conditions in the decision making processes.

In this paper we present some issues to study and to guarantee consistency in the decision making problems under fuzzy preference relations. We give a new characterization of fuzzy consistency based on the additive transitivity property which facilitates the verification of consistency in the case of fuzzy preference relations. Using this new characterization we present a method to construct consistent fuzzy preference relations from $n-1$ given preference values. Additionally, making use of the transformation function that relates multiplicative preference relations and fuzzy preference relations [3] we develop a similar study of consistency in the case of multiplicative preference relations $[11,12]$.

In order to do that, the paper is organized as follows. Section 2 presents the use of the preference relations in decision making. Section 3 studies the different characterizations of consistency of fuzzy preference relations. Section 4 defines a new characterization of consistency and the constructing method of consistent fuzzy preference relations. Finally, some concluding remarks are pointed out in Section 5.

\section{The use of the preference relations in decision making}

Preference relation is the most common representation of information used in decision making problems because it is a useful tool in modelling decision processes, above all when we want to aggregate experts' preferences into group preferences $[6,11,12,14,16]$. In a preference relation an expert associates to every pair of alternatives a value that reflects some degree of preference of the first alternative over the second one. Many important decision models have been developed using mainly two kinds of preference relations:

- Multiplicative preference relations [11, 12]: A multiplicative preference relation $A$ on a set of alternatives $X$ is represented by a matrix $A \subset X \mathrm{x} X, A=\left(a_{i j}\right)$, being $a_{i j}$ interpreted as the ratio of the preference intensity of alternative $x_{i}$ to that of $x_{j}$, i.e., it is interpreted as $x_{i}$ is $a_{i j}$ times as good as $x_{j}$. According to Miller's study [10], Saaty suggests measuring $a_{i j}$ using a ratio scale, and precisely the 1 to 9 scale $[11,12]: a_{i j}=1$ indicates indifference between $x_{i}$ and $x_{j}, a_{i j}=9$ indicates that $x_{i}$ is absolutely preferred to $x_{j}$, and $a_{i j} \in\{1, \ldots, 9\}$ indicates intermediate preference evaluations. In this case, the preference relation, $A$, is usually assumed multiplicative reciprocal, i.e.,

$$
a_{i j} \cdot a_{j i}=1 \quad \forall i, j \in\{1, \ldots, n\} .
$$

Saaty means by consistency what he calls cardinal transitivity in the strength of preferences which is a stronger condition than the traditional requirement of the transitivity of preferences. Thereby, the definition of consistency proposed by Saaty is the following [11, 12] 
Definition 2.1. A reciprocal multiplicative preference relation $A=\left(a_{i j}\right)$ is consistent if

$$
a_{i j} \cdot a_{j k}=a_{i k} \quad \forall i, j, k=1, \ldots, n \text {. }
$$

- Fuzzy preference relations [2, 6, 15]: A fuzzy preference relation $P$ on a set of alternatives $X$ is a fuzzy set on the product set $X \times X$, that is characterized by a membership function

$$
\mu_{P}: X \times X \longrightarrow[0,1] .
$$

When cardinality of $X$ is small, the preference relation may be conveniently represented by the $n \times n$ matrix $P=\left(p_{i j}\right)$ being $p_{i j}=\mu_{P}\left(x_{i}, x_{j}\right) \quad \forall i, j \in\{1, \ldots, n\} . p_{i j}$ is interpreted as the preference degree of the alternative $x_{i}$ over $x_{j}: p_{i j}=1 / 2$ indicates indifference between $x_{i}$ and $x_{j}\left(x_{i} \sim x_{j}\right), p_{i j}=1$ indicates that $x_{i}$ is absolutely preferred to $x_{j}$, and $p_{i j}>1 / 2$ indicates that $x_{i}$ is preferred to $x_{j}\left(x_{i} \succ x_{j}\right)$. In this case, the preference matrix, $P$, is usually assumed additive reciprocal, i.e.,

$$
p_{i j}+p_{j i}=1 \quad \forall i, j \in\{1, \ldots, n\} .
$$

In [3] we studied the transformation function between reciprocal multiplicative preference relations with values in the interval scale $[1 / 9,9]$ and reciprocal fuzzy preference relations with values in $[0,1]$. This study can be summarized in the following proposition.

Proposition 2.1. Suppose that we have a set of alternatives, $X=\left\{x_{1}, \ldots, x_{n}\right\}$, and associated with it a reciprocal multiplicative preference relation $A=\left(a_{i j}\right)$ with $a_{i j} \in[1 / 9,9]$. Then, the corresponding reciprocal fuzzy preference relation, $P=\left(p_{i j}\right)$ with $p_{i j} \in[0,1]$, associated with $A$ is given as follows:

$$
p_{i j}=g\left(a_{i j}\right)=\frac{1}{2} \cdot\left(1+\log _{9} a_{i j}\right) .
$$

With such a transformation function $g$ we can relate the research issues obtained for both kinds of preference relations.

In this paper, we focus on the consistency of the decision models based on the fuzzy preference relations. In the following section, we study briefly the different proposals to characterize consistency of the fuzzy preference relations existing in the literature.

\section{On consistency of the fuzzy preference relations}

For making a consistent choice when assuming fuzzy preference relations a set of consistency properties to be satisfied by such relations have been suggested. Transitivity is one of the most important properties concerning preferences, and it represents the idea that the preference value obtained by comparing directly two alternatives should be equal to or greater than the preference value between those two alternatives obtained using an indirect chain of alternatives $[5,9,15]$. Some of the suggested properties are given:

1. Triangle condition [9]: $p_{i j}+p_{j k} \geq p_{i k} \quad \forall i, j, k$.

This condition can be geometrically interpreted considering alternatives $x_{i}, x_{j}, x_{k}$ as the vertices of a triangle with length sides $p_{i j}, p_{j k}$ and $p_{i k}$ [9], and therefore the length corresponding to the vertices $x_{i}, x_{k}$ should not exceed the sum of the lengths corresponding to the vertices $x_{i}, x_{j}$ and $x_{j}, x_{k}$. 
2. Weak transitivity [15]: $p_{i j} \geq 0.5, p_{j k} \geq 0.5 \Rightarrow p_{i k} \geq 0.5 \forall i, j, k$.

The interpretation of this condition is the following: If $x_{i}$ is preferred to $x_{j}$ and $x_{j}$ is preferred to $x_{k}$, then $x_{i}$ should be preferred to $x_{k}$. This kind of transitivity is the usual transitivity condition that a logical and consistent person should use if he/she does not want to express inconsistent opinions, and therefore it is the minimum requirement condition that a consistent fuzzy preference relation should verify.

3. Max-min transitivity [5, 17]: $p_{i k} \geq \min \left(p_{i j}, p_{j k}\right) \forall i, j, k$.

The idea represented here is that the preference value obtained by a direct comparison between two alternatives should be equal to or greater than the minimum partial values obtained when comparing both alternatives with an intermediate one. This kind of transitivity has been the traditional requirement to characterize consistency in the case of fuzzy preference relations [17], although it is a very strong concept that it could not be verified even when a fuzzy preference relation is considered perfectly consistent from a practical point of view. For example, let us consider a set of three alternatives $X=\left\{x_{1}, x_{2}, x_{3}\right\}$, such that $x_{1} \prec x_{2} \prec x_{3}$. Suppose that the opinions about these alternatives are given by the following reciprocal fuzzy preference relation

$$
P=\left(\begin{array}{ccc}
0.5 & 0.1 & 0 \\
0.9 & 0.5 & 0.4 \\
1 & 0.6 & 0.5
\end{array}\right)
$$

On the one hand, this matrix reflects the fact that $x_{1} \prec x_{2} \prec x_{3}$; it verifies weak transitivity and the triangle condition. On the other hand, it does not verifies max-min transitivity because $p_{13}<\min \left\{p_{12}, p_{23}\right\}$.

4. Max-max transitivity [5, 17]: $p_{i k} \geq \max \left(p_{i j}, p_{j k}\right) \quad \forall i, j, k$.

This concept represents the idea that the preference value obtained by a direct comparison between two alternatives should be equal to or greater than the maximum partial values obtained when comparing both alternatives using an intermediate one. This is a stronger concept than max-min transitivity and therefore if a reciprocal fuzzy preference relation does not verify the latter neither verifies the former.

5. Restricted max-min transitivity [15]: $p_{i j} \geq 0.5, p_{j k} \geq 0.5 \Rightarrow p_{i k} \geq \min \left(p_{i j}, p_{j k}\right) \quad \forall i, j, k$.

When a fuzzy preference relation verifies this condition it is modelled the concept that when an alternative $x_{i}$ is preferred to $x_{j}$ with a value $p_{i j}$ and $x_{j}$ is preferred to $x_{k}$ with a value $p_{i j}$, then $x_{i}$ should be preferred to $x_{k}$ with at least an intensity of preference $p_{i k}$ equal to the minimum of the above values. The inequality should becomes equality only when there exist indifference between at least two of the three alternatives. A consistent fuzzy preference relation has to verify this condition, which goes a step further than weak transitivity because add an extra requirement about the degrees of preferences involved. This transitivity condition is therefore stronger than weak transitivity but it is milder than max-min transitivity. It is easy to prove that the above fuzzy reciprocal preference relation $P$ verifies restricted max-min transitivity.

6. Restricted max-max transitivity [15]: $p_{i j} \geq 0.5, p_{j k} \geq 0.5 \Rightarrow p_{i k} \geq \max \left(p_{i j}, p_{j k}\right) \quad \forall i, j, k$.

In this case it is modelled the concept that when an alternative $x_{i}$ is preferred to $x_{j}$ with a value $p_{i j}$ and $x_{j}$ is preferred to $x_{k}$ with a value $p_{i j}$, then $x_{i}$ should be preferred to $x_{k}$ with at least an intensity of preference $p_{i k}$ equal to the maximum of the above values. As in the previous case, the the equality should hold only when there exist indifference between 
at least two of the three alternatives, in which case, restricted max-max transitivity and restricted max-min transitivity coincide. It is clear that this concept is, on the one hand, stronger than restricted max-min transitivity and, on the other hand, milder than max-max transitivity. This concept has been considered by Tanino [15] as a compulsory condition to be verified by a consistent fuzzy preference relation. It is easy to prove that the fuzzy reciprocal preference relation $P$, given above, verifies restricted max-max transitivity.

7. Multiplicative transitivity [15]: $\frac{p_{j i}}{p_{i j}} \cdot \frac{p_{k j}}{p_{j k}}=\frac{p_{k i}}{p_{i k}} \quad \forall i, j, k$.

Tanino in [15] introduced this concept of transitivity only in the case of being $p_{i j}>0 \forall i, j$, and interpreting $p_{i j} / p_{j i}$ as a ratio of the preference intensity for $x_{i}$ to that of $x_{j}$, i.e., $x_{i}$ is $p_{i j} / p_{j i}$ times as good as $x_{j}$. Multiplicative transitivity includes restricted max-max transitivity [14, 15], and rewritten as $p_{i j} \cdot p_{j k} \cdot p_{k i}=p_{i k} \cdot p_{k j} \cdot p_{j i} \forall i, j, k$ can be extended to the whole set of reciprocal fuzzy preference relations, i.e., when values of $p_{i j}$ can be equal to 0 .

8. Additive transitivity [14, 15]: $\left(p_{i j}-0.5\right)+\left(p_{j k}-0.5\right)=\left(p_{i k}-0.5\right) \forall i, j, k$, or equivalently $p_{i j}+p_{j k}+p_{k i}=\frac{3}{2} \quad \forall i, j, k$.

This type of transitivity has the following interpretation: suppose we do want to establish a ranking between three alternatives $x_{i}, x_{j}$, and $x_{k}$. If we do not have any information about these alternatives it is natural to start assuming that we are in an indifference situation, that is, $x_{i} \sim x_{j} \sim x_{k}$, and therefore when giving preferences this situation is represented by $p_{i j}=p_{j k}=p_{i k}=0.5$. Suppose now that we have a piece of information that says alternative $x_{i} \prec x_{j}$, that is $p_{i j}<0.5$. It is clear then that $p_{j k}$ or $p_{k i}$ have to change otherwise there would be a contradiction because we would have $x_{i} \prec x_{j} \sim x_{k} \sim x_{i}$. If we suppose that $p_{j k}=0.5$ then we have the situation: $x_{j}$ is preferred to $x_{i}$ and there is no difference in preferring $x_{j}$ to $x_{k}$. We must conclude then that $x_{k}$ has to be preferred to $x_{i}$. Furthermore, as $x_{j} \sim x_{k}$ then $p_{j i}=p_{k i}$, and so $p_{i j}+p_{j k}+p_{k i}=p_{i j}+p_{j k}+p_{j i}=1+0.5=1.5$. We have the same conclusion if $p_{k i}=0.5$. In the case of being $p_{j k}<0.5$, then we have that $x_{k}$ is preferred to $x_{j}$ and this to $x_{i}$, so $x_{k}$ should be preferred to $x_{i}$. On the other hand, the value $p_{k i}$ has to be equal to or greater than $p_{j i}$, being equal only in the case of $p_{j k}=0.5$ as we have seen. Interpreting the value $p_{j i}-0.5$ as the intensity of preference of alternative $x_{j}$ over $x_{i}$, then it seem reasonable to suppose that the intensity of preference of $x_{k}$ over $x_{i}$ should be equal to the sum of the intensities of preferences when using an intermediate alternative $x_{j}$, that is $p_{k i}-0.5=\left(p_{k j}-0.5\right)+\left(p_{j i}-0.5\right)$. The same reasoning can be applied in the case of $p_{j k}>0.5$. The reciprocal fuzzy preference relation $P$, given above, verifies additive transitivity. It is easy to prove that additive transitivity is a stronger concept than restricted max-max transitivity $[14,15]$.

From the above list, the additive transitivity seems to be an acceptable property to characterize consistency in the case of fuzzy preference relations given that: The weak transitivity is the minimum requirement condition that a consistent fuzzy preference relation should verify. The max-min transitivity has been the traditional requirement to characterize consistency in the case of fuzzy preference relations. The max-max transitivity is a stronger concept than maxmin transitivity. Both transitivity concepts are too strong in the sense that they could not be verified even when a fuzzy preference relation is considered perfectly consistent from a practical point of view (as was shown in the above example). Restricted max-min and restricted maxmax transitivity concepts seem good alternatives to them, being restricted max-max transitivity even more adequate from a practical point of view than restricted max-min transitivity; moreover, restricted max-max transitivity implies restricted max-min transitivity. The multiplicative 
transitivity concept is valid only in the case of being $p_{i j}>0 \forall i, j$. The additive transitivity is a stronger concept than restricted max-max transitivity and it implies restricted max-max transitivity. If we want to include a some kind of measure of strength of preference in the concept of transitivity then additive transitivity includes this idea of ordinal strength of preferences. Furthermore, as it is shown in the next result, the consistency definition in the case of the multiplicative preference relations via the above transformation function $g$ (given in proposition 2.1 ) is equivalent to the additive transitivity property.

Proposition 3.1. Let $A=\left(a_{i j}\right)$ be a consistent multiplicative preference relation, then the corresponding reciprocal fuzzy preference relation, $P=g(A)$ verifies additive transitivity property.

Proof. For being $A=\left(a_{i j}\right)$ consistent we have that $a_{i j} \cdot a_{j k}=a_{i k} \forall i, j, k$, or equivalently $a_{i j} \cdot a_{j k} \cdot a_{k i}=1 \forall i, j, k$. Taking logarithms on both sides, we have

$$
\log _{9} a_{i j}+\log _{9} a_{j k}+\log _{9} a_{k i}=0 \quad \forall i, j, k .
$$

Adding 3 and dividing by 2 both sides then

$$
\frac{1}{2} \cdot\left(1+\log _{9} a_{i j}\right)+\frac{1}{2} \cdot\left(1+\log _{9} a_{j k}\right)+\frac{1}{2} \cdot\left(1+\log _{9} a_{k i}\right)=\frac{3}{2} \quad \forall i, j, k .
$$

The fuzzy preference relation $P=g(A)$, being $p_{i j}=\frac{1}{2} \cdot\left(1+\log _{9} a_{i j}\right)$, verifies

$$
p_{i j}+p_{j k}+p_{i k}=\frac{3}{2} \forall i, j, k
$$

We conclude that $P=g(A)$ verifies additive transitivity property.

In such a way, in this paper we consider the following definition of the consistent fuzzy preference relation:

Definition 3.1. A reciprocal fuzzy preference relation $P=\left(p_{i j}\right)$ is consistent if

$$
p_{i j}+p_{j k}+p_{k i}=\frac{3}{2} \forall i, j, k=1, \ldots, n .
$$

In what follows, we will use the term additive consistency to refer to consistency for fuzzy preference relations based on the additive transitivity property.

\section{Additive consistency}

In this section we present a new characterization of the additive consistency condition given by definition 3.1, which states that for checking additive consistency of a fuzzy preference relation $\mathrm{P}$, it is only necessary to check those triplets of values $(i, j, k)$ verifying $i \leq j \leq k$. As a consequence of this equivalent condition, we design a method to construct consistent fuzzy preference relations from a set of n-1 preference values which guarantees consistency of the fuzzy preference relations provided by the experts. We conclude this section by exporting the above research issues on additive consistency to the multiplicative decision models [11, 12], i.e., decision models based on multiplicative preference relations. 


\subsection{Characterization of additive consistency}

Proposition 4.1. For a reciprocal fuzzy preference relation $P=\left(p_{i j}\right)$, the following statements are equivalent:

i) $p_{i j}+p_{j k}+p_{k i}=\frac{3}{2} \quad \forall i, j, k$.

ii) $p_{i j}+p_{j k}+p_{k i}=\frac{3}{2} \quad \forall i<j<k$.

Proof. $i) \Rightarrow$ ii). Obvious.

ii) $\Rightarrow i$ ). It is clear that if two of the indexes $i, j, k$ are equal, or the three of them, $i$ ) reduces to the reciprocity condition which we are assuming. Therefore, we have to prove that condition $i)$ is verified for indexes $i, j, k$ taking different values. There are six possible cases:

Case $1 . i<j<k$. In this case $i$ ) reduces to $i i$ ), and therefore $i$ ) is true.

Case 2. $i<k<j$. Using reciprocity property and the fact that it is verified that $p_{i k}+p_{k j}+p_{j i}=$ $\frac{3}{2}$,

$$
p_{i j}+p_{j k}+p_{k i}=1-p_{j i}+1-p_{k j}+1-p_{i k}=3-\left(p_{i k}+p_{k j}+p_{j i}\right)=3-\frac{3}{2}=\frac{3}{2}
$$

Case 3. $j<i<k$. We have that $p_{j i}+p_{i k}+p_{k j}=\frac{3}{2}$, and by reciprocity property:

$$
p_{i j}+p_{j k}+p_{k i}=1-p_{j i}+1-p_{k j}+1-p_{i k}=3-\left(p_{j i}+p_{i k}+p_{k j}\right)=3-\frac{3}{2}=\frac{3}{2}
$$

Case 4. $j<k<i . p_{i j}+p_{j k}+p_{k i}=p_{j k}+p_{k i}+p_{i j}=\frac{3}{2}$

Case 5. $k<i<j . p_{i j}+p_{j k}+p_{k i}=p_{k i}+p_{i j}+p_{j k}=\frac{3}{2}$

Case 6. $k<j<i$. In this case $p_{j i}+p_{i k}+p_{k j}=\frac{3}{2}$, and using reciprocity property, we have that:

$$
p_{i j}+p_{j k}+p_{k i}=1-p_{j i}+1-p_{k j}+1-p_{i k}=3-\left(p_{k j}+p_{j i}+p_{i k}\right)=3-\frac{3}{2}=\frac{3}{2} .
$$

Proposition 4.1 can be rewritten as follows:

Proposition 4.2. A fuzzy preference relation $P=\left(p_{i j}\right)$ is consistent if and only if

$$
p_{i j}+p_{j k}+p_{i k}=\frac{3}{2}, \quad \forall i \leq j \leq k .
$$

Proof. We only have to demonstrate that $p_{i j}+p_{j k}+p_{k i}=\frac{3}{2}, \quad \forall i \leq j \leq k$ implies that $P$ is reciprocal.

Let $i<j$, we will show firstly that $p_{i i}=\frac{1}{2}, \forall i$, and secondly that $p_{i j}+p_{j i}=1, \forall i, j$. If we take $i=j=k$ then we have that:

$$
p_{i i}+p_{i i}+p_{i i}=\frac{3}{2}
$$

and therefore

$$
p_{i i}=\frac{1}{2}, \quad \forall i
$$

On the other hand, if we take $j=k$, then

$$
p_{i j}+p_{j i}=p_{i j}+p_{j j}+p_{j i}-\frac{1}{2}=\frac{3}{2}-\frac{1}{2}=1 .
$$


The following result characterizes additive consistency.

Proposition 4.3. For a reciprocal fuzzy preference relation $P=\left(p_{i j}\right)$, the following statements are equivalent:

i) $p_{i j}+p_{j k}+p_{k i}=\frac{3}{2} \quad \forall i<j<k$,

ii) $p_{i(i+1)}+p_{(i+1)(i+2)}+\ldots+p_{(j-1) j}+p_{j i}=\frac{j-i+1}{2} \quad \forall i<j$.

Proof. $i) \Rightarrow$ ii). Let $i<j$ and $k=j-i$. The expression ii) can be rewritten as follows:

$$
p_{i(i+1)}+p_{(i+1)(i+2)}+\ldots+p_{(i+k-1)(i+k)}+p_{(i+k) i}=\frac{k+1}{2} \quad \forall i<j .
$$

We will use mathematical induction to prove this part of the proposition. The base clause it is obviously true for $k=1$, because it is reduced to the reciprocity property, which we are assuming. The recursion clause requires us to show that if the hypothesis is true for $k=n$

$$
p_{i(i+1)}+p_{(i+1)(i+2)}+\ldots+p_{(i+n-1)(i+n)}+p_{(i+n) i}=\frac{n+1}{2}
$$

then it is true for $k=n+1$ :

$$
\begin{aligned}
p_{i(i+1)}+\ldots+p_{(i+n)(i+n+1)}+p_{(i+n+1) i} & =\left(p_{i(i+1)}+\ldots+p_{(i+n-1)(i+n)}\right)+p_{(i+n)(i+n+1)}+p_{(i+n+1) i} \\
& =\left(\frac{n+1}{2}-p_{(i+n) i}\right)+p_{(i+n)(i+n+1)}+p_{(i+n+1) i} \\
& =\frac{n-1}{2}+p_{i(i+n)}+p_{(i+n)(i+n+1)}+p_{(i+n+1) i} \\
& =\frac{n-1}{2}+\frac{3}{2}=\frac{n+2}{2}
\end{aligned}
$$

so the result is established.

ii) $\Rightarrow i$ ). Let $i<j<k$

$$
\begin{aligned}
p_{i j}+p_{j k}+p_{k i} & =1-p_{j i}+1-p_{k j}+p_{k i} \\
& =2+\left(p_{i(i+1)}+\ldots+p_{(j-1) j}-\frac{j-i+1}{2}\right)+\left(p_{j(j+1)}+\ldots+p_{(k-1) k}-\frac{k-j+1}{2}\right)+p_{k i} \\
& =\frac{2-k+i}{2}+\left(p_{i(i+1)}+\ldots+p_{(k-1) k}+p_{k i}\right) \\
& =\frac{2-k+i}{2}+\frac{k-i+1}{2} \\
& =\frac{3}{2} .
\end{aligned}
$$

\subsection{A method to construct consistent fuzzy preference relations}

The result presented in proposition 4.3 is very important because it can be used to construct a consistent fuzzy preference relation from the set of $n-1$ values $\left\{p_{12}, p_{23}, \ldots, p_{n-1 n}\right\}$. In such a way, we can facilitate experts the expression of consistent preferences in the decision processes. 
Example 1. Suppose that we have a set of four alternatives $\left\{x_{1}, x_{2}, x_{3}, x_{4}\right\}$ where we have certain knowledge to assure that alternative $x_{1}$ is weakly more important than alternative $x_{2}$, alternative $x_{2}$ is more important than $x_{3}$ and finally alternative $x_{3}$ is strongly more important than alternative $x_{4}$. Suppose that this situation is modelled by the preference values $\left\{p_{12}=0.55, p_{23}=0.65, p_{34}=0.75\right\}$. Applying proposition 4.3, we obtain:

$$
\begin{aligned}
& p_{31}=1.5-p_{12}-p_{23}=1.5-0.55-0.65=0.3, \\
& p_{41}=2-p_{12}-p_{23}-p_{34}=2-0.55-0.65-0.75=0.05, \\
& p_{42}=1.5-p_{23}-p_{34}=1.5-0.65-0.75=0.1, \\
& p_{21}=1-p_{12}=0.45, p_{13}=1-p_{31}=0.7, p_{14}=1-p_{41}=0.95, \\
& p_{32}=1-p_{23}=0.35, p_{24}=1-p_{42}=0.9, p_{43}=1-p_{34}=0.25,
\end{aligned}
$$

and therefore:

$$
P=\left(\begin{array}{cccc}
0.5 & 0.55 & 0.7 & 0.95 \\
0.45 & 0.5 & 0.65 & 0.9 \\
0.3 & 0.35 & 0.5 & 0.75 \\
0.05 & 0.1 & 0.25 & 0.5
\end{array}\right)
$$

We make note that, if the primary values are different then we would have obtained a matrix $P$ with entries not in the interval $[0,1]$, but in an interval $[-a, 1+a]$, being $a>0$. In such a case, we would need to transform the obtained values using a transformation function which preserves reciprocity and additive consistency, that is a function $f:[-a, 1+a] \longrightarrow[0,1]$, verifying

1. $f(-a)=0$.

2. $f(1+a)=1$.

3. $f(x)+f(1-x)=1, \forall x \in[-a, 1+a]$.

4. $f(x)+f(y)+f(z)=\frac{3}{2}, \forall x, y, z \in[-a, 1+a]$ such that $x+y+z=\frac{3}{2}$.

The linear solution verifying 1 and 2 takes the form $f(x)=\varphi \cdot x+\beta$, being $\varphi, \beta \in \mathbb{R}$. This function is

$$
f(x)=\frac{1}{1+2 a} \cdot x+\frac{a}{1+2 a}=\frac{x+a}{1+2 a}
$$

which verifies 3

$$
f(x)+f(1-x)=\frac{x+a}{1+2 a}+\frac{1-x+a}{1+2 a}=\frac{x+a+1-x+a}{1+2 a}=1
$$

and when $x+y+z=\frac{3}{2}$

$$
f(x)+f(y)+f(z)=\frac{x+a}{1+2 a}+\frac{y+a}{1+2 a}+\frac{z+a}{1+2 a}=\frac{x+y+z+3 a}{1+2 a}=\frac{3 / 2+3 a}{1+2 a}=\frac{3}{2}
$$

verifies 4

Summarizing: The method to construct a consistent reciprocal fuzzy preference relation $P^{\prime}$ on $X=\left\{x_{1}, \ldots, x_{n}, n \geq 2\right\}$ from $n-1$ preference values $\left\{p_{12}, p_{23}, \ldots, p_{n-1 n}\right\}$ presents the following steps: 
1. Compute the set of preference values $B$ as

$$
\begin{aligned}
& B=\left\{p_{i j}, i<j \wedge p_{i j} \notin\left\{p_{12}, p_{23}, \ldots, p_{n-1 n}\right\}\right\} \\
& p_{i j}=\frac{j-i+1}{2}-p_{i i+1}-p_{i+1 i+2} \ldots-p_{j-1 j} .
\end{aligned}
$$

2. $a=\left|\min \left\{B \cup\left\{p_{12}, p_{23}, \ldots, p_{n-1 n}\right\}\right\}\right|$

3. $P=\left\{p_{12}, p_{23}, \ldots, p_{n-1 n}\right\} \cup B \cup\left\{1-p_{12}, 1-p_{23}, \ldots, 1-p_{n-1 n}\right\} \cup \neg B$.

4. The consistent fuzzy preference relation $P^{\prime}$ is obtained as $P^{\prime}=f(P)$ such that

$$
\begin{gathered}
f:[-a, 1+a] \longrightarrow[1,0] \\
f(x)=\frac{x+a}{1+2 a} .
\end{gathered}
$$

\subsection{Exporting the issues on additive consistency to the multiplicative deci- sion models}

In this subsection we apply the above issues on additive consistency in the decision models based on multiplicative preference relations by means of the transformation function $g$ given in section 2. We use the term multiplicative consistency to refer to consistency for multiplicative preference relations. Thereby, we state an equivalent condition to multiplicative consistency given by definition 2.1, which states that for checking multiplicative consistency it is only necessary to check those triplets of values $(i, j, k)$ verifying $i<j<k$. Then, as a consequence, we design a method to construct consistent multiplicative preference relations from a set of n-1 preference values.

Proposition 4.4. For a reciprocal multiplicative preference relation $A=\left(a_{i j}\right)$, the following statements are equivalent:

i) $a_{i j} \cdot a_{j k}=a_{i k} \quad \forall i, j, k$.

ii) $a_{i j} \cdot a_{j k}=a_{i k} \quad \forall i<j<k$.

Proposition 4.4 can be rewritten as follows:

Proposition 4.5. A multiplicative preference relation $A=\left(a_{i j}\right)$ is consistent if and only if $a_{i j} \cdot a_{j k}=a_{i k}, \quad \forall i \leq j \leq k$.

The following result characterizes the multiplicative consistency.

Proposition 4.6. For a reciprocal multiplicative preference relation $A=\left(a_{i j}\right)$, the following statements are equivalent:

i) $a_{i j} \cdot a_{j k}=a_{i k} \quad \forall i<j<k$.

ii) $a_{i j}=a_{i i+1} \cdot a_{i+1 i+2} \ldots \cdot a_{j-1 j} \quad \forall i<j$.

The above propositions reduce to the propositions 4.1, 4.2, 4.3, using the transformation function $g$, respectively.

The result presented in proposition 4.6 can be used to construct a consistent multiplicative preference relation from the set of n- 1 values $\left\{a_{12}, a_{23}, \ldots, a_{n-1 n}\right\}$. In such a way, we can facilitate experts the expression of consistent preferences in the multiplicative decision processes. 
Example 2. Suppose that we have a set of four alternatives $\left\{x_{1}, x_{2}, x_{3}, x_{4}\right\}$ where we have certain knowledge to assure that alternative $x_{1}$ is weakly more important than alternative $x_{2}$, alternative $x_{2}$ is strongly more important than $x_{3}$ and finally alternative $x_{3}$ is very strongly more important than alternative $x_{4}$. Applying Saaty's scale this situation is represented by the set of values $\left\{a_{12}=3, a_{23}=5, a_{34}=7\right\}$, and therefore when constructing the consistent multiplicative preference relation we have that:

$$
\begin{aligned}
& a_{13}=a_{12} \cdot a_{23}=3 \cdot 5=15, \\
& a_{14}=a_{12} \cdot a_{23} \cdot a_{34}=3 \cdot 5 \cdot 7=105, \\
& a_{24}=a_{23} \cdot a_{34}=5 \cdot 7=35, \\
& a_{21}=1 / a_{12}=1 / 3, a_{31}=1 / a_{13}=1 / 15, a_{41}=1 / a_{14}=1 / 105, \\
& a_{32}=1 / a_{23}=1 / 5, a_{42}=1 / a_{24}=1 / 35, a_{43}=1 / a_{34}=1 / 7,
\end{aligned}
$$

and the consistent multiplicative preference relation obtained is the following:

$$
A=\left(\begin{array}{cccc}
1 & 3 & 15 & 105 \\
1 / 3 & 1 & 5 & 35 \\
1 / 15 & 1 / 5 & 1 & 7 \\
1 / 105 & 1 / 35 & 1 / 7 & 1
\end{array}\right)
$$

The problem here, as we can observe, is that the ratio scale for comparison $[1 / 9,9]$ is not preserved, as we get values greater that 9 . Nevertheless, we can always assure that the values we get will belong to a ratio scale of the form $[1 / a, a]$. At this point, if we want to preserve the use of the scale $[1 / 9,9]$ we do have to transform the values using a transformation function which preserves reciprocity and consistency, that is a function $f:[1 / a, a] \longrightarrow[1 / 9,9]$ verifying

1. $f(a)=9$,

2. $f(x) \cdot f(1 / x)=1, \forall x \in[1 / a, a]$, and

3. $f(x) \cdot f(y)=f(z), \forall x, y, z \in[1 / a, a]$ such that $x \cdot y=z$.

With property 2 we assure that function $f$ maintains reciprocity, while property 3 means that function $f$ preserves multiplicative consistency.

In property 2 , if we take the particular value $x=1$ we have that $f(1)=1$, and therefore this property can be rewritten as follows: $f(x) \cdot f(1 / x)=f(1)$, which implies

1. $f(x) \cdot f(1 / x) \cdot f(y) \cdot f(1 / y)=1 \cdot 1=1$,

2. $f(x \cdot y) \cdot f(1 /(x \cdot y))=1$,

that is

$$
f(x \cdot y) \cdot f(1 /(x \cdot y))=f(x) \cdot f(1 / x) \cdot f(y) \cdot f(1 / y),
$$

and by symmetry, only one of the following four functional equations is true [7]:

1. $f(x \cdot y)=f(x) \cdot f(y) \forall x, y \in[1 / a, a]$.

2. $f(x \cdot y)=f(x) \cdot f(1 / y) \forall x, y \in[1 / a, a]$.

3. $f(x \cdot y)=f(1 / x) \cdot f(y) \forall x, y \in[1 / a, a]$.

4. $f(x \cdot y)=f(1 / x) \cdot f(1 / y) \forall x, y \in[1 / a, a]$. 
We are going to demonstrate that the only possible functional equation is the first one.

1. If we suppose that $f(x \cdot y)=f(x) \cdot f(1 / y)$, then from property 2 we have that $f(1 / y)=$ $1 / f(y)$ and therefore $f(x \cdot y)=f(x) / f(y) \forall x, y \in[1 / a, a]$. Taking the value $x=1$ we have that $f(y)=1 / f(y) \forall y \in[1 / a, a]$, which implies that $f(y)=1 \forall y \in[1 / a, a]$.

2. If $f(x \cdot y)=f(1 / x) \cdot f(y)$ then $f(x \cdot y)=1 /(f(x) \cdot f(y)) \forall x, y \in[1 / a, a]$. In this case we have for $y=1$ that $f(x)=1 / f(x) \forall x \in[1 / a, a]$, which implies that $f(x)=1 \forall x \in[1 / a, a]$.

3. If $f(x \cdot y)=f(1 / x) \cdot f(1 / y)$ then $f(x \cdot y)=f(x) / f(y) \forall x, y \in[1 / a, a]$. For $x=1$ we have that $f(y)=1 / f(y) \forall y \in[1 / a, a]$, which implies again that $f(y)=1 \forall y \in[1 / a, a]$.

We conclude that in cases 2,3 and 4 the one possible solution is $f(y)=1 \forall y \in[1 / a, a]$, which contradicts property 1 . So, function $f$ verifies the first relation, i.e.,

$$
f(x \cdot y)=f(x) \cdot f(y) \forall x, y \in[1 / a, a] .
$$

It is well-known that the general solution of this functional equation is $f(x)=x^{c}, c>0[1]$. From $f(1)=9$, it results that the function we are looking for is

$$
f(x)=x^{1 / \log _{9} a} .
$$

We note that we did not use property 3 to obtain function $f$, although it is verified

$$
\begin{gathered}
\forall x, y, z \in[1 / a, a] \text { such that } x \cdot y=z \Rightarrow(x \cdot y)^{1 / \log _{9} a}=z^{1 / \log _{9} a} \Rightarrow \\
x^{1 / \log _{9} a} \cdot y^{1 / \log _{9} a}=z^{1 / \log _{9} a} \Rightarrow f(x) \cdot f(y)=f(z) .
\end{gathered}
$$

Example 3. Applying function $f$ to the above consistent multiplicative preference relation $A$ with values in $[1 / 105,105]$ we have the also consistent multiplicative preference relation $f(A)=A^{\prime}$ with approximated values in $[1 / 9,9]$

$$
A^{\prime}=\left(\begin{array}{cccc}
1 & 42 / 25 & 18 / 5 & 9 \\
25 / 42 & 1 & 107 / 50 & 134 / 25 \\
5 / 18 & 50 / 107 & 1 & 5 / 2 \\
1 / 9 & 25 / 134 & 2 / 5 & 1
\end{array}\right) .
$$

Summarizing: The method to construct a consistent multiplicative preference relation $A^{\prime}$ on $X=\left\{x_{1}, \ldots, x_{n}, n \geq 2\right\}$ from n-1 preference values $\left\{a_{12}, a_{23}, \ldots, a_{n-1 n}\right\}$ presents the following steps:

1. Compute the set of preference values $B$ as

$$
\begin{gathered}
B=\left\{a_{i j}, i<j \wedge a_{i j} \notin\left\{a_{12}, a_{23}, \ldots, a_{n-1 n}\right\}\right\} \\
a_{i j}=a_{i i+1} \cdot a_{i+1 i+2} \ldots \cdot a_{j-1 j} .
\end{gathered}
$$

2. $a=\max B$

3. $A=\left\{a_{12}, a_{23}, \ldots, a_{n-1 n}\right\} \cup B \cup\left\{a_{12}, a_{23}, \ldots, a_{n-1 n}\right\}^{-1} \cup B^{-1}$.

4. The consistent multiplicative preference relation $A^{\prime}$ is obtained as $A^{\prime}=f(A)$ such that

$$
\begin{gathered}
f:\left[\frac{1}{a}, a\right] \longrightarrow\left[\frac{1}{9}, 9\right] \\
f(x)=x^{1 / \log _{9} a} .
\end{gathered}
$$




\section{Concluding remarks}

We have studied consistency conditions to be verified in the decision making processes in the case of working with fuzzy preference relations. We have given a new characterization of consistency based on the additive transitivity property of fuzzy preference relations. This new characterization of consistency allows us to check easily the consistency in the experts' opinions. Furthermore, we have presented a method to construct consistent preference relations by using a set of specific $n-1$ preference data obtained by comparing an alternative only just with the next one. In such a way, we have presented a proposal to facilitate to the experts the expression of consistent opinions in order to overcome the inconsistencies that can appear in the decision processes based on fuzzy preference relations. Finally, we have shown that this consistency study of fuzzy preference relations is exportable to the case of multiplicative preference relations via the transformation function presented in [3].

In the future, we will focus on the second problem of consistency in decision making, i.e., when a whole group of experts are considered consistent $[4,8]$.

\section{References}

[1] J. Aczél, Lectures on Functional Equations and Their Applications (Academic Press, New York, 1966).

[2] F. Chiclana, F. Herrera, E. Herrera-Viedma, Integrating Three Representation Models in Fuzzy Multipurpose Decision Making Based on Fuzzy Preference Relations, Fuzzy Sets and Systems 97 (1998) 33-48.

[3] F. Chiclana, F. Herrera, E. Herrera-Viedma, Integrating Multiplicative Preference Relations in a Multipurpose Decision Making Model Based on Fuzzy Preference Relations, Fuzzy Sets and Systems 112 (2001) 277-291.

[4] V. Cutello and J. Montero, Fuzzy Rationality Measures, Fuzzy Sets and Systems 62 (1994) 39-54.

[5] D. Dubois, H. Prade, Fuzzy Sets and Systems: Theory and Application, (Academic Press, New York, 1980).

[6] J. Fodor, M. Roubens, Fuzzy Preference Modelling and Multicriteria Decision Support (Kluwer, Dordrecht, 1994).

[7] F. Herrera, E. Herrera-Viedma, F. Chiclana, Multiperson Decision Making Based on Multiplicative Preference Relations, European Journal of Operational Research 129 (2001) $372-385$.

[8] F. Herrera, E. Herrera-Viedma, J.L. Verdegay, A Rational Consensus Model in Group Decision Making using Linguistic Assessments, Fuzzy Sets and Systems 88 (1997) 31-49.

[9] R.D. Luce, P. Suppes, Preferences, Utility and Subject Probability. In: R.D. Luce et al., Eds., Handbook of Mathematical Psychology, Vol. III, (Wiley, New York, 1965) 249-410.

[10] G. A. Miller, The Magical Number Seven or Minus Two: Some Limits on Our Capacity of Processing Information, Psychological Rev. 63 (1956) 81-97.

[11] Th. L. Saaty, The Analytic Hierarchy Process (McGraw-Hill, New York, 1980). 
[12] Th. L. Saaty, Fundamentals of Decision Making and Priority Theory with the AHP (RWS Publications, Pittsburgh, 1994).

[13] A. K. Sen, Social Choice Theory: A Re-Examination, Econometrica 45 (1977) 53-89.

[14] T. Tanino, Fuzzy Preference Orderings in Group Decision Making. Fuzzy Sets and Systems 12 (1984) 117-131.

[15] T. Tanino, Fuzzy Preference Relations in Group Decision Making. In: J. Kacprzyk, M. Roubens (Eds.), Non-Conventional Preference Relations in Decision Making (SpringerVerlag, Berlin, 1988) 54-71.

[16] E. Triantaphyllou, Multi-Criteria Decision Making Methods: A Comparative Study (Kluwer Academic Publishers, Dordrecht, 2000).

[17] H.-J. Zimmermann, Fuzzy Set Theory and Its Applications (Kluwer, Dordrecht, 1991). 